Original Article

\title{
Validity of body impedance analysis for evaluating body composition in patients undergoing long-term hemodialysis
}

\author{
Masahiro Noguchi, PT, MS ${ }^{1)^{*}}$, Shinichi Yamaguchi, PT ${ }^{2)}$, Yoshitaka Koshino, MD $^{3)}$, Akira \\ Kimura, PT, PhD ${ }^{4}$, Shigeji Miyagi, PhD $^{5}$ ) \\ 1) Faculty of Health Science, Kinjo University: 1200 Kasama-machi, Hakusan City, Ishikawa \\ 924-8511, Japan \\ 2) Department of Physical Therapy, Moriyama Koshino Clinic, Japan \\ 3) Department of Internal Medicine, Mizuho Hospital, Japan \\ 4) Faculty of Health Science, Gunma Paz College, Japan \\ 5) Department of Health Administration, Kagawa Nutrition University, Japan
}

\begin{abstract}
Purpose] This study assessed changes in body composition before and after dialysis in chronic hemodialysis patients and determined the relationships between various body composition parameters and blood lipid levels in these patients. [Subjects] The cross-sectional study included 19 dialysis outpatients (17 men and 2 women, aged 35-82 years). [Methods] Body mass index, body weight, percent body fat, and percent skeletal muscle were measured before and after dialysis by using body impedance analysis. Blood lipid levels were obtained from patients' clinical records. The body composition parameters before and after dialysis were compared using paired t-tests. Spearman's rank correlation coefficients were calculated to determine relationships between the body composition parameters, before and after dialysis, and the blood lipid levels. [Results] All body composition parameters differed significantly before and after dialysis. High-density lipoprotein cholesterol level significantly correlated with all the body composition parameters, whereas total cholesterol, low-density lipoprotein cholesterol, and triglyceride levels significantly correlated with some of these parameters. The correlation coefficients revealed no major differences in the relationships between blood lipid parameters and body compositions before and after dialysis. [Conclusion] Our findings suggest that body composition parameters, whether measured before or after dialysis, can be used to evaluate obesity in longitudinal studies.

Key words: Dialysis, Body composition, Blood lipid
\end{abstract}

(This article was submitted Jan. 5, 2015, and was accepted Feb. 7, 2015)

\section{INTRODUCTION}

In recent years, the incidence of obesity-related glomerulopathy has been steadily increasing in patients with chronic kidney disease (CKD). According to one report, the relative risks for $\mathrm{CKD}$ in overweight (body mass index [BMI] of $25 \mathrm{~kg} / \mathrm{m}^{2}$ or higher) and obese individuals (BMI of $30 \mathrm{~kg} / \mathrm{m}^{2}$ or higher), as compared to individuals with a normal BMI $\left(18-25 \mathrm{~kg} / \mathrm{m}^{2}\right)$, are 1.40 and 1.83 respectively ${ }^{1)}$. Another study reported the independent risk ratio for CKD as 1.86 in patients with metabolic syndrome ${ }^{2}$.

Therefore, we believe that education on obesity prevention is important for patients with renal failure, especially during the early stages of the renal disease. Moreover, once

*Corresponding author. Masahiro Noguchi (E-mail: noguchi@kinjo.ac.jp)

C2015 The Society of Physical Therapy Science. Published by IPEC Inc. This is an open-access article distributed under the terms of the Creative Commons Attribution Non-Commercial No Derivatives (by-ncnd) License $<$ http://creativecommons.org/licenses/by-nc-nd/3.0/> . dialysis is initiated in such patients, there is a simultaneous need to address cardiovascular risk factors in these patients in order to reduce the overall risk of mortality. Currently, various anti-obesity measures are being initiated, and some of these involve estimation of body fat. Numerous methods are available for estimating body fat, including dual-energy $\mathrm{x}$-ray absorptiometry, computed tomography, magnetic resonance imaging, underwater weighing method, and body impedance analysis (BIA). Body composition analyzers, used in BIA, are low-priced and allow measurement of body fat without any assistance from a medical specialist.

In BIA, a low electrical current is introduced into the body. Lean tissue with high water content offers low resistance, while fat tissue with low water content offers high resistance to electric current; therefore, electrical current can pass more easily through lean tissue than fat tissue. Impedance is the frequency-subordinate resistance of a conductor to exchange a current. It is determined by resistance $(\mathrm{R})$ and reactance $(\mathrm{Xc}) . \mathrm{R}$ is the pure resistance of a conductor to a current. Xc is the amount of accumulation of electricity of the electric charge produced by capacitors, such as a cell membrane and an interface section between organizations. 
Bioelectric impedance $(Z)$, measured in ohms, is the square root of the sum of the squares of $\mathrm{R}$ and $\mathrm{Xc}^{3}$ ).

As BIA involves estimation using a low electrical current, the amount of body water plays an important role in this analysis - if the instrument is precise, data measured after bath, voiding, drinking, etc., might result in an error. Notably, patients with CKD remove excess body water through dialysis, as their kidneys are unable to effectively filter body fluids and waste products. Therefore, the amount of body water changes significantly before and after dialysis in these patients. The amount of percent body fat (\%BF) estimated by BIA in patients with renal failure undergoing dialysis might differ from the true values on account of retention of atypical amounts of water in these patients. Thus, the question arises which body composition parameters, whether those measured before dialysis or after dialysis, should preferably be used in health assessment of dialysis patients.

To the best of our knowledge, there are some studies that report the relationship between body composition and effect of exercise in dialysis patients or in patients with other arteriosclerotic diseases, such as stroke ${ }^{4,5)}$; however, thus far, no study has specifically investigated the validity of body composition parameters, before and after dialysis, in health assessment of dialysis patients. With this in mind, the purpose of the present study was to assess the changes in body composition parameters before and after dialysis in chronic hemodialysis patients and to determine the strengths of linear relationships between various body composition parameters and blood lipid levels in these patients.

\section{SUBJECTS AND METHODS}

The participants in this study were 19 Japanese outpatients ( 17 men and 2 women; mean \pm SD age, $54 \pm 12$ years; range, 35-82 years) attending a dialysis center in June 2013. This research complies with the terms of the Declaration of Helsinki. The research protocol was explained to the participants, who subsequently provided their informed consent, prior to the commencement of the study. Moreover, additional prior information was provided to the participants, including explanations about freedom of research participation, protection of personal data, and freedom to withdraw from the study even after providing the informed consent. These explanations were given verbally and in writing to the participants. All participants signed a letter of consent. Patients unable to stand during measurements were excluded, as this was a basic requirement of the study. In addition, patients having serious dementia, patients on pacemakers, and cancer patients were excluded from the study. In two subjects, the indication for dialysis was obesity-related diabetic renal disease.

We performed a cross-sectional experimental study, which compared body composition parameters before and after dialysis, and determined the strengths of linear relationships among body composition parameters, blood lipid levels, and physical activity (PA). We measured body composition parameters, including body weight (BW), BMI, \%BF, and percent skeletal muscle (\%SM), using an HBF-362 body composition monitor (Omron Healthcare, Kyoto, Japan). Physical therapists interviewed the subjects
Table 1. Changes in body composition parameters before and after dialysis in chronic hemodialysis patients $(n=19)$ as assessed by body impedance analysis

\begin{tabular}{lcc}
\hline & Pre-dialysis & Post-dialysis \\
\hline BW $(\mathrm{kg})$ & $63.6 \pm 9.2$ & $61.8 \pm 8.9^{* *}$ \\
BMI $\left(\mathrm{kg} / \mathrm{m}^{2}\right)$ & $23.4 \pm 3.3$ & $22.7 \pm 3.2^{* *}$ \\
$\% \mathrm{BF}(\%)$ & $18.4 \pm 8.4$ & $22.0 \pm 8.5^{* *}$ \\
$\% \mathrm{SM}(\%)$ & $32.3 \pm 4.3$ & $30.5 \pm 4.2^{* *}$ \\
\hline
\end{tabular}

All data are presented as mean \pm SD

BW: body weight; BMI: body mass index; \%BF: percent body fat; \%SM: percent skeletal muscle

$* * \mathrm{p}<0.01$

to record their physical activities during the last 24 hours. Based on the type and intensity of these activities, levels of PA were calculated for each of the participants ${ }^{6,7)}$. PA was assessed in all the participants on the day of dialysis as well as on a non-dialysis day. The blood lipid profile included total cholesterol (TC), high-density lipoprotein cholesterol (HDL-C), low-density lipoprotein cholesterol (LDL-C), and triglyceride (TG) levels obtained from the patients' clinical records.

The body composition analyzer used in the study was located near the entrance of the dialysis room. The body composition parameters in each of the participants were measured before the dialysis, and again after the dialysis session, which lasted for 3-4 hours. The blood lipid levels were measured every month, and we adopted the latest available data for evaluating their relationships with the body composition parameters.

We evaluated normality of data using the Shapiro-Wilk test. As a result, the normality of dialysis PA and TG levels could not be verified. Therefore, body composition before and after dialysis was compared using parametric tests, and the rest of the statistical analyses were conducted using nonparametric tests. The body composition parameters before and after dialysis were compared by using paired t-tests. Spearman's rank correlation coefficients were calculated to determine relationships among body composition parameters, blood lipid levels, and PA. All statistical analyses were performed using SPSS version 19.0 software (IBM, Armonk, NY, USA). The level of statistical significance was set at $5 \%$.

\section{RESULTS}

The changes in body composition parameters before and after dialysis are shown in Table 1 . All body composition parameters differed significantly before and after dialysis ( $\mathrm{p}$ $<0.01)$. BW, BMI, and \%SM were found to be significantly lower, and $\% \mathrm{BF}$ was found to be significantly higher after dialysis than those before dialysis.

The relationships (in terms of Spearman's rank correlation coefficients) between various body composition parameters and blood lipid levels before and after dialysis are shown in Table 2. HDL-C, BMI, \%BF, and \%SM before dialysis were found to be strongly correlated with blood lipid levels. Strong correlations were also observed in case of body composition parameters measured after dialysis. 
Table 2. The relationships (in terms of Spearman's rank correlation coefficients) between various body composition parameters and blood lipid levels in chronic hemodialysis patients $(\mathrm{n}=19)$

\begin{tabular}{lccccc}
\hline & & TC & HDL-C & LDL-C & TG \\
\hline Pre-dialysis & BW & 0.320 & $-0.532^{*}$ & 0.432 & $0.479^{*}$ \\
& BMI & 0.309 & $-0.598^{* *}$ & $0.505^{*}$ & 0.377 \\
& $\% \mathrm{BF}$ & $0.582^{* *}$ & $-0.711^{* *}$ & $0.788^{* *}$ & $0.551^{*}$ \\
& $\% \mathrm{SM}$ & $-0.498^{*}$ & $0.582^{* *}$ & $-0.685^{* *}$ & -0.373 \\
Post-dialysis & $\mathrm{BW}$ & 0.318 & $-0.681^{* *}$ & 0.415 & $0.494^{*}$ \\
& $\mathrm{BMI}$ & 0.319 & $-0.593^{* *}$ & $0.501^{*}$ & 0.386 \\
& $\% \mathrm{BF}$ & $0.620^{* *}$ & $-0.681^{* *}$ & $0.789^{* *}$ & $0.651^{* *}$ \\
& $\% \mathrm{SM}$ & $-0.539^{*}$ & $0.571^{*}$ & $-0.709^{* *}$ & -0.429 \\
\hline
\end{tabular}

TC: total cholesterol; HDL-C: high-density lipoprotein cholesterol; LDL-C: low-density lipoprotein cholesterol; TG: triglyceride; BW: body weight; BMI: body mass index; \%BF: percent body fat; \%SM: percent skeletal muscle $* \mathrm{p}<0.05, * * \mathrm{p}<0.01$

The mean PA on the day of dialysis was found to be significantly lower than the PA on a non-dialysis day; however, the correlation coefficients were not significant among body composition parameters, blood lipid levels, and PA.

\section{DISCUSSION}

Renal replacement therapy comprises dialysis and renal transplantation. In Japan, dialysis is more common. Dialysis involves filtration, performed by the kidneys in normal individuals, by an external dialyzer. It removes waste products and excess water and electrolytes. In general, dialysis is performed 2 or 3 times a week. As patients under dialysis cannot remove the accumulated body water for about 2 days, they tend to have excess body water before dialysis. Conversely, after dialysis, the amount of body water is drastically reduced.

In this study, we found a higher $\% \mathrm{BF}$ and a lower \%SM after dialysis than before dialysis. This suggests that the observed differences in \%BF and \%SM before and after dialysis resulted from changes in the amount of body water. Measurements using the body composition analyzer are made after information regarding age, sex, and height of a subject is entered into the apparatus. First, the weight of the subject is measured using the body composition analyzer. Thereafter, a small current is sent through the subject's body - impedance is measured and \% $\mathrm{BF}$ is calculated. Because age, sex, and height remain constant before and after dialysis, the changes in BW and impedance solely influence the estimated $\% \mathrm{BF}$. These changes, in turn, can be directly attributed to the changes in the amount of body water.

Increases in fat mass, especially visceral fat, result in increased free fatty acid secretion from the adipose tissue. As a result, large quantities of free fatty acids accumulate in the liver, causing hepatic lipogenesis. Simultaneously, free fatty acids in the blood also increase ${ }^{8,9}$. Kobayashi and colleagues reported that the $\% \mathrm{BF}$ estimated by $\mathrm{BIA}$ is useful for the prediction of TC, LDL-C, and TG levels in Japanese men $^{10)}$. Consequently, increases in \%BF have been related to worsening of blood lipid parameters. In this study, we examined the validity of these relationships.
Our analyses revealed strong correlations between body composition parameters and blood lipid levels. Thus, our study supports the view that there are strong correlations between \%BF and blood lipid levels in dialysis patients, and that the measurement of $\% \mathrm{BF}$ has relevance in the context of health assessment in dialysis patients. In fact, we originally hypothesized that post-dialysis data would be more useful for this health assessment as the amount of body water would have been regulated by dialysis. However, no major differences were observed in the strengths of the relationships between the body composition parameters, before and after dialysis, and the blood lipid levels. This was corroborated by strong correlation between these variables both before and after dialysis. We conclude that body composition parameters including \%BF, whether measured before or after dialysis, might be useful in health assessment of dialysis patients.

There were a number of limitations in the present study. We examined the relationships between the body composition parameters and the blood lipid levels to verify the usefulness of body composition parameters in health assessment of dialysis patients. However, body fat distribution was reported to be strongly correlated with other data. Therefore, further studies need to investigate the relevance of data on coronary artery disease risk factors, such as the arteriosclerosis index and blood glucose levels, along with that of body composition data in the context of usefulness in health assessment of patients, thus probably contributing towards better health outcomes. Moreover, we need to focus attention on fat distribution and to evaluate the accumulation of visceral fat by modalities such as magnetic resonance imaging or computed tomography. In this study, out of the various body composition parameters, we paid particular attention to the $\% \mathrm{BF}$, and tried to verify the body composition of dialysis patients by studying the relationship with the blood lipid levels. However, the reliability of body composition parameters after dialysis, and the reliability of BIA in dialysis patients need to be further studied. An additional limitation of the present study was that the subjects included only two women. Therefore, the influence of gender cannot be ruled out. In general, women tend to have higher levels of 
body fat and fewer skeletal muscle cells than men. Besides, the subjects in our study ranged widely in age. However, we consider the overall effects of gender and age to be minimal. Thus, all the subjects were included in the final analysis. Nevertheless, to get a better insight into this issue, studies focused on only one gender or specific age groups need to be conducted in the future. Lastly, this study was a crosssectional study; although our results suggest that the body composition parameters before and after dialysis might have applications in health assessment of chronic hemodialysis patients, further large-scale longitudinal studies should be performed to confirm our findings.

\section{REFERENCES}

1) Wang Y, Chen X, Song Y, et al.: Association between obesity and kidney disease: a systematic review and meta-analysis. Kidney Int, 2008, 73: 19-33. [Medline] [CrossRef]

2) Tozawa $\mathrm{M}$, Iseki $\mathrm{C}$, Tokashiki $\mathrm{K}$, et al.: Metabolic syndrome and risk of developing chronic kidney disease in Japanese adults. Hypertens Res, 2007, 30: 937-943. [Medline] [CrossRef]

3) Baumgartner RN, Chumlea WC, Roche AF: Bioelectric impedance phase angle and body composition. Am J Clin Nutr, 1988, 48: 16-23. [Medline] 4) Cho H, Sohng KY: The effect of a virtual reality exercise program on physical fitness, body composition, and fatigue in hemodialysis patients. J Phys Ther Sci, 2014, 26: 1661-1665. [Medline] [CrossRef]

5) Hirayama M, Nagata $Y$, Tsutou A: Relationship between body composition and functional outcomes in males with subacute stroke. J Phys Ther Sci, 2007, 19: 177-182. [CrossRef]

6) Kimura A: Validity of a new method for the estimation of energy expenditure based on position, perceived intensity and duration in college students. J Jpn Phys Ther Assoc, 2004, 31: 147-154 (in Japanese).

7) Kimura A: Validity of a new method to estimate energy consumption of daily physical activities over a 24 hour period in a group of elderly subjects with motor disturbance. J Jpn Phys Ther Assoc, 2004, 31: 160-167 (in Japanese).

8) Kuriyama H, Yamashita S, Shimomura I, et al.: Enhanced expression of hepatic acyl-coenzyme A synthetase and microsomal triglyceride transfer protein messenger RNAs in the obese and hypertriglyceridemic rat with visceral fat accumulation. Hepatology, 1998, 27: 557-562. [Medline] [CrossRef]

9) Vecchini A, Ceccarelli V, Orvietani P, et al.: Enhanced expression of hepatic lipogenic enzymes in an animal model of sedentariness. J Lipid Res, 2003, 44: 696-704. [Medline] [CrossRef]

10) Kobayashi J, Murano S, Kawamura I, et al.: The relationship of percent body fat by bioelectrical impedance analysis with blood pressure, and glucose and lipid parameters. J Atheroscler Thromb, 2006, 13: 221-226. [Medline] [CrossRef] 